\title{
THE SINGULARITIES NEAR THE CORNER OF A VISCOELASTIC FLUID IN A 2D CAVITY
}

\author{
Hüseyin Demir* \\ R. W. Williams** \\ F. T. Akyildiz* \\ * The University of Ondokuz Mayis, Science and Art Faculty, Dept. of Mathematics, \\ Samsun / Turkey \\ ** The University of Glamorgan, Dept. of Maths \& Computing, Treeforest, Wales / U.K
}

\begin{abstract}
In this article finite differences are used to study viscoelastic incompressible flow of a Criminale Erickson Filbey fluid in a square cavity flow domain. In this case, the nature of corner singularities is examined in which the fluid is contained and the flow generated by the motion of one or more walls. The governing equations are formulated in terms of stream function and vorticity equation and the corresponding radial parts are defined by a fourth-order non-linear differential equations for Stokes flow. In recent years that mathematical formulations of viscoelastic flows often remain very complex velocity and stress field and then stress singularities are known to occur in several flows as in this article. Therefore, singularity behaviour became a very important current issue in fluid dynamics. However, this article is set up with the aim of examining the corner singularities for cavity driven flow in $2 \mathrm{D}$ for viscoelastic flow despite the Newtonian flow being well known. Then we show that the viscoelastic fluid has different singularity behaviour than the viscous thid near the comer with respect to the shear-rate.
\end{abstract}

\section{INTRODUCTION}

The corner singularity problem of the non-Newtonian flow in flow domain has been subject of experimental and numerical study for over 20 years [1] and is a valuable work in fluid dynamics. These techniques were first used by Dean and Mantagnon [2] and later developments include the work of Moffat [3]. Moreover, Davies [4] described the methods of how an investigation of the effects of elasticity on eddies could be made. Because the local behaviour of flow variables in two-dimensional Stokes flow has proved the existence of such eddies in wedges and non-re-entrant corners. Many important studies were carried out but first study was done by Walters and Webster [5]. They reported that corner conditions had a greater effect to the flow in the case of non-Newtonian flows than corresponding Newtonian flows in their experiments. Some disadvantages can be expected for this problem for nonNewtonian flows than their Newtonian counterparts in numerical studies due to numerical discretison errors occurs near the corner. This can be supported by the nature of the governing equations which are being different type partial differential equations in each case. For example, while the non-linear partial differential governing equations for non-Newtonian flows are of mixed elliptic-hyperbolic type for steady incompressible flows, the corresponding Newtonian flows are elliptic. Therefore, this problem gives undesirable results in the simulation of non-Newtonian flows, apart from a few works [6] in which special methods were used to define boundary singularity behaviour which can be existed near the corner, in velocity gradients, stress and pressure instead of using the numerical techniques [4] 
In standard cavity flow, singularities exist near the top two corners because the shear-rate becomes increasingly large and therefore both shear-rate and vorticity become singular

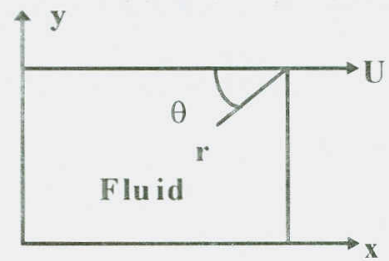

Figure 1. Diagram of the corner singularity

To consider the standard cavity flow whose top plate moves with constant speed (I we assume that no-slip boundary conditions are applied. We next examine the nature of the corner singularities separately for both Newtonian and non-Newtonian flow.

\section{THE CORNER SINGULARITIES OF VISCOUS FLOW}

\subsection{Newtonian Flow}

In steady two-dimensional viscous incompressible flow the stream function and vorticity equations are

$$
\begin{gathered}
\nabla^{2} \omega=\operatorname{Re} \frac{D \omega}{D t} \\
\nabla^{2} \Psi=\omega
\end{gathered}
$$

Moffat [2] has shown that the viscous forces dominate the inertial forces near the corner itself. Then equation (1) becomes

$$
\nabla^{2} \omega=0
$$

and equation (2) and (3) give to rise to

$$
\nabla^{4} \Psi=0
$$

Equation (4) is called the Stokesian flow equation.

Considering the polar co-ordinates $(r, \theta)$ with origin at one singular corner the stream function takes the form as

$$
\Psi(r, \theta)=r^{m} f_{m}(\theta)
$$

where only the solution for $m=1$ is allowed due to the boundary conditions.

In this case the vorticity and shear-rate near the corner are given by

$$
\omega=\frac{1}{r} g(\theta)
$$

and

$$
\dot{\gamma}=\frac{1}{r} h(\theta)
$$

where $g(\theta)$ and $h(\theta)$ incorporate $f_{1}(\theta)$ and its derivatives. As seen from (6) and (7), when $r \rightarrow 0$ the vorticity and shear-rate become infinite and singularities exist at the corner points. 
Similarly by using equation (4) in terms of polar co-ordinates and on using (5), we have the general form of $f_{m}(\theta)$ as

$$
f_{m}(\theta)=A \cos m \theta+B \sin m \theta+C \cos (m-2) \theta+D \sin (m-2) \theta
$$

where $\mathrm{A}, \mathrm{B}, \mathrm{C}$ and $\mathrm{D}$ are constants. In the special case $\mathrm{m}=1$ the solution takes the form (which is for the Stokesian flow equation)

$$
f_{1}(\theta)=A \cos \theta+B \sin \theta+C \theta \cos \theta+D \theta \sin \theta .
$$

The boundaries may be rigid walls on which the velocity is defined, or surfaces on which the stress is defined. Therefore, for standard cavity flow with one wall moving and the other stationary we have

$$
\left.\begin{array}{l}
f_{1}(0)=0, \\
f_{1}\left(\frac{\pi}{2}\right)=0, \\
\frac{d}{d \theta} f_{1}(0)=U, \\
\frac{d}{d \theta} f_{1}\left(\frac{\pi}{2}\right)=0
\end{array}\right\}
$$

where $\theta=0$ and $\theta=\frac{\pi}{2}$ represent moving wall and stationary wall respectively. The solution of the Stokesian flow equation near the corner is

$$
\begin{gathered}
\Psi(r, \theta)=\frac{r I I}{\left(\pi^{2}-4\right)}\left(-\pi^{2} \sin \theta+4 \theta \cos \theta+2 \pi \sin \theta\right) \\
\omega(r, \theta)=\frac{-4 U}{r\left(\pi^{2}-4\right)}(\pi \cos \theta-2 \sin \theta)
\end{gathered}
$$

\subsection{Non-Newtonian Flow}

To use Moffat's assumption, we need to see how inelastic non-Newtonian flow behaves near the corner. In this case the flow equation is

$$
\eta \nabla^{2} \omega+2\left(\frac{\partial}{\partial x} \eta \frac{\partial}{\partial x} \omega+\frac{\partial}{\partial y} \eta \frac{\partial}{\partial y} \omega\right)+M(\eta) M(\Psi)+L(\eta) L(\Psi)=0
$$

where $\mathrm{M}$ and $\mathrm{L}$ are defined as before. Equation (13) is usually solved with equation (2) We consider the viscosity near the corner through the Cross-model for non-Newtonian viscous flow. Since we work with polar co-ordinates the velocity components are defined in terms of polar co-ordinates by 


$$
\begin{aligned}
& u(\theta)=-\left(s f+c \frac{\partial f}{\partial \theta}\right) \\
& v(\theta)=c f-s \frac{\partial f}{\partial \theta}
\end{aligned}
$$

where $f=f_{1}(\theta), s=\sin \theta$ and $c=\cos \theta$. The Cross-model viscosity, therefore, takes the form

$\eta=a+b(\theta) r^{1-n}$, as $r \rightarrow 00<\mathrm{n}<1$, where $a=\eta(\infty)$.

When we substitute the stream, vorticity and viscosity function, are defined in terms of polar co-ordinates, into (13) the equation of the non-Newtonian flow near the corner is

$$
\left(a+b(\theta) r^{1-n}\right) \nabla^{2} \omega+C(\theta) r^{-n-2}=0 .
$$

Here $C^{\prime}(\theta)$ incorporates terms including $f(\theta)$ since $\omega$ has the form $\omega=\frac{1}{r} g(\theta), \quad \nabla^{2} \omega$ assumes the form

$$
\nabla^{2} \omega=\frac{1}{r^{3}} D(\theta)
$$

Therefore, we can write for equation (15)

$$
\nabla^{2} \omega\left\{1+\frac{C(\theta) r^{-n-2}}{\left(a+b(\theta) r^{1-n}\right) r^{-3} D(\theta)}\right\}=0 .
$$

Finally we obtain

$$
\nabla^{2} \omega\left\{1+\frac{C(\theta) r^{1-n}}{\left(a+E(\theta) r^{1-n}\right) L(\theta)}\right\}=0
$$

When $r \rightarrow 0, \nabla^{2} \omega \rightarrow 0$ and $\nabla^{4} \Psi \rightarrow 0$. We can therefore say that the flow near the corner is Stokesian for shear-dependent non-Newtonian viscous flow with these approximations.

\subsection{The Corner Singularities of Viscoelastic Fluids}

The flow of viscoelastic fluids in regions involving high stresses, it shows the different behaviour from the viscous flow near the singularity. It is a matter of fact that in nonNewtonian flows little is known about local behaviour near the corner apart from a few cases [4].

We use a similar analysis as before and examine the steady viscoelastic fluid behaviour near the corner, for simplicity, whose model is denoted by CEF. It appears likely that the corner singularity of the viscoelastic fluids may create significant numerical problems. We assume as previously the Moffat's assumption that the viscous force still dominate the inertial force as $\mathrm{Re} \rightarrow 0$. Under these circumstances, for the viscoelastic fluid 


$$
\eta^{2} H(\eta ; \omega)+M(\Psi) M(\eta)+L(\Psi) L(\eta)-\frac{1}{2} M\left(S_{x x}-S_{\eta \gamma}\right)-L\left(S_{x y}\right)=0
$$

where $\mathrm{H}, \mathrm{M}$ and $\mathrm{L}$ are defined as before

Near the corner, we use a similar analysis to that used earlier to examine the singularity behaviour.

When the polar forms for $\Psi, \omega, \eta$ are substituting into (19) we have

$$
\left(a+b(\theta) r^{1-n}\right) \nabla^{2} \omega+A(\theta) r^{-n-2}+B(\theta) r^{-n-3}=0
$$

Here $A(\theta)$ and $B(\theta)$ depend on $f(\theta)$ since

$$
\nabla^{2} \omega=\frac{1}{r^{3}} D(\theta)
$$

we have

$$
\nabla^{2} \omega\left\{1+\left[\frac{A(\theta)+B(\theta) r^{-1}}{\left(a+E(\theta) r^{1-n}\right) D(\theta)}\right] r^{1-n}\right\}=0
$$

where $\mathrm{B}(\theta)$ represents the elastic part of the viscoealstic flow equation and

$$
B(\theta)=(n+1)(n+2) F_{1}(\theta)+(n+2) F_{2}(\theta)+(n+1) F_{3}(\theta)+F_{4}(\theta)
$$

Here $F_{1}(\theta), F_{2}(\theta), F_{3}(\theta)$ and $F_{4}(\theta)$ involves too many functions of $\theta$ to incorporate have and we omit their full expression. For $n=1$ equation (22) takes the form

$$
B(\theta)=6 F_{1}(\theta)+3 F_{2}(\theta)+2 F_{3}(\theta)+F_{4}(\theta)
$$

If $B(\theta)=0$ and $0 \leq n \leq 1$, then we may say from equation (21) that the viscoelastic fluid behaviour near the corner is Stokesian as is its counterpart Newtonian fluid

Then we can say that the well known Giesekus-Tanner theorem for second order fluids $(n=1)$ is consistent with the Moffat's assumption which has been used to examine the flow behaviour near the corner earlier i.e. $B(\theta)=0$ for $n=1$ in equation (21).

If the Moffat's assumption is true for all $n$ close to 1 (where $n<1$ ), we require $B(\theta)=0$.

We obtain the equation

$$
(n-1)\left[(n+4) F_{1}(\theta)+F_{2}(\theta)+F_{3}(\theta)\right]=0
$$

by subtracting the right hand side of equation (23) (which must now be zero) from the right hand side of equation (22) and assuming $B(\theta)=0$ for $n<1$.

In our analysis we find $F_{1}(\theta) \neq 0$, therefore $B(\theta) \neq 0$ for all $n<1$, whereas $B(\theta)=0$ for $n=1$.

In this case when viscosity is not a constant there may be elastic domination near the corner invalidating the Moffat's assumption. This is a problem for future study. In conclusion we can 
say that the flow is Stokesian near the corner for shear dependent Newtonian and nonNewtonian viscous flow. Under same circumstance the fluid is Stokesian near the comci for the viscoelastic fluid with constant viscosity but the problem remains many questions unanswered for the flow with variab!e viscosity.

\section{CONCLUSION}

The corner singularity in the 2D flow was investigated. It was determined that flow near the corner singularity for the shear dependent Newtonian and non-Newtonian purely viscous flows can be regarded as Stokesian flow. When the same analysis was undertaken for the viscoelastic fluids it was found that they can behave as Stokesian flow near the corner for the constant viscosity case. However the problem remains unresolved for variable viscosity fluids.

\section{REFERENCES}

1. M. J. Crochet, A. R. Davýes and K. Walters, Numerical simulation of non-Newtonian flow Rheology Series 1, Elsevier, 1984

2. W. R. Dean and P. E. Montagnon, Proc. Camb. Phil. Soc., 45, 389-394, 1949.

3. H. K. Moffat, J. Fluid Mech., 18, 1-18, 1964.

4. A. R. Davies, Reentrant corner singularities in non-Newtonian flow, 29, 269-293, 1988

5. K. W. Walters and M.F. Webster, Phil. Trans. R. Soc. London Ser. A., 308, 192-218, 198 ?

6. A. R. Davies, S. J. Lee and M. F. Webster, J. Non-Newtonian Fluid Mech., 16, 117-139 1984 